\title{
Data Analysis of Infection Rates Among Exposed Healthcare Workers Could Reveal Effective Prophylactics Against SARS-CoV-2
}

\author{
Guido Núñez-Mujica, Therese Kichuk, \\ and César Carrasco-López
}

\section{Abstract}

The global crisis provoked by the SARS-CoV2 pandemic, and the economic and social consequences associated to the essential policies applied to contain it, necessitates the expedited development of therapeutic solutions. It is a priority to produce data both rapidly and accurately in order to identify current therapies that can be repurposed to offer protection from SARS-CoV-2 infection. As healthcare workers are both at high risk for infection and able to be readily diagnosed, they offer a potential wealth of data to be analyzed. A systematic data analysis of exposure and infection rates among healthcare workers could yield patterns identifying common protective factors, such as medications with prophylactic potential against SARS$\mathrm{CoV}-2$, that can be fast-tracked into available

\section{G. Núñez-Mujica}

In Situ Diagnostics, Santiago, Chile

GenoSUR, Miami, FL, USA

T. Kichuk

Department of Molecular Biology, Princeton University, Princeton, NJ, USA

C. Carrasco-López $(\bowtie)$

Department of Chemical and Biological Engineering,

Princeton University, Princeton, NJ, USA

e-mail: cesarc@princeton.edu therapies. With results suggesting their activity against other coronaviruses and their widespread adoption, Antiretroviral cocktails could be a promising initial target for such large-scale data analysis approach.

\section{Keywords}

Antiretroviral · Antiviral · Coronavirus . COVID-19 $\cdot$ Data science $\cdot$ Healthcare .

Vaccine

The social distancing measures being implemented worldwide have proven to be effective in slowing the spread of the coronavirus SARS-CoV-2 (Ji et al. 2020). The devastating economic and societal consequences of these measures (Lippi et al. 2020; Cornwall 2020), however, could make them unsustainable and challenge their extended implementation throughout the process of developing, approving, and widely administering a vaccine. We are faced with a situation in which long-term enforcement of practices employed to minimize the deadly consequences of this pandemic could result in political, social and economic catastrophe- especially for our most vulnerable populations (Cornwall 2020). Therefore, it is of vital importance to accelerate the discovery of effective 
prophylactics to aid in safely ramping down social distancing policies.

In order to accelerate the identification of useful compounds, it is crucial to investigate repurposing medications already approved to combat disease in clinical settings. Numerous clinical trials have been rapidly initiated to test the efficacy of currently available drugs in the treatment of SARS-CoV-2 (U. S. National Library of Medicine, Clinical Trials System $2020 a$, b). It is necessary, however, to also increase the number of trials assessing the efficacy of these medications in preventing the infection, as studies performed only with patients who are seriously ill might lead researchers to erroneously reject compounds that could offer protection against the virus at earlier stages.

There are several proposals to initiate clinical trials aiming to study the prophylactic effects of compounds such as hydroxychloroquine and ritonavir/lopinavir (U. S. National Library of Medicine, Clinical Trials System 2020c). To our knowledge, however, there are no systematic approaches based on large scale data analysis to identify compounds that could prevent infection with SARS-CoV-2. Within each hospital, there is a population of healthcare workers who offer the potential for such analysis. As healthcare workers are at the front line in the battle against this pandemic, they are both at high risk for infection and able to be readily diagnosed. Systematic analysis of exposure and infection rates among healthcare workers could yield patterns identifying common protective factors such as medications with prophylactic potential against SARS-CoV-2. With this approach relying on previously approved medications, if a compound were to exhibit strong protective effects, it could be quickly tested and adopted to prevent SARS-CoV-2 infections in healthcare workers and older adults. A prophylactic antiviral against SARS-CoV-2 with prior approval for clinical use could accelerate the restoration of social and economic activity and ease the strain that essential policies have placed on the most vulnerable of the world's citizens until an effective vaccine is ready for widespread distribution.
While novel investigations and treatments are critical in the study of any disease, the urgency provoked by this pandemic calls for data to be generated both rapidly and accurately. It is a priority to identify current therapies that can be repurposed to offer protection from SARS-CoV2 infection and there is a wealth of data to analyze in hospitals with known cases. Assembling study cohorts of healthcare workers based on medication usage could rapidly reveal differential infection rates and aid efforts to fast-track therapies. Given results suggesting activity of antiretroviral cocktails against SARS and MERS (Chu et al. 2004; Chan et al. 2015), the widespread adoption of these therapies and observations of lower SARS-CoV-2 infections in HIV+ patients in South East Asia (Joob and Wiwanitkit 2020), the protective impact of antiretroviral medications could be a promising initial subject for a large scale data analysis approach.

\section{References}

Chan J, Yao Y, Yeung ML, Deng W, Bao L, Jia L, Li F, Xiao C, Gao H, Yu P, Cai JP, Chu H, Zhou J, Chen H, Qin C, Yuen K (2015) Treatment with Lopinavir/ritonavir or interferon- $\beta 1 \mathrm{~b}$ improves outcome of MERS$\mathrm{CoV}$ infection in a nonhuman primate model of common marmoset. J Infect Dis 212(12):1904-1913. https://doi.org/10.1093/infdis/jiv392

Chu C, Cheng V, Hung I, Wong M, Chan K, Chan KS, Kao R, Poon L, Wong C, Guan Y, Peiris J, Yuen K (2004) Role of lopinavir/ritonavir in the treatment of SARS: initial virological and clinical findings. Thorax 59(3):252-256. https://doi.org/10.1136/thorax.2003. 012658

Cornwall W (2020) Can you put a price on COVID-19 options? Experts weigh lives versus economics. Science. https://doi.org/10.1126/science.abb9969

Ji T, Chen H, Xu J, Wu L, Li J, Chen K, Qin G (2020) Lockdown contained the spread of 2019 novel coronavirus disease in Huangshi city, China: early epidemiological findings. Clin Infect Dis. https://doi.org/10. 1093/cid/ciaa390

Joob B, Wiwanitkit V (2020) SARS-CoV-2 and HIV. J Med Virol. https://doi.org/10.1002/jmv.25782. [Epub ahead of print]

Lippi G, Henry B, Bovo C, Sanchis-Gomar F (2020) Health risks and potential remedies during prolonged lockdowns for coronavirus disease 2019 (COVID-19). Diagnosi. https://doi.org/10.1515/dx-2020-0041

U. S. National Library of Medicine, Clinical Trials System (2020a) Hydroxychloroquine vs. azithromycin for 
hospitalized patients with suspected or confirmed COVID-19 (HAHPS) NCT04329832. Created on 04/04/2020, Accessed on 16 Apr 2020. https://www. clinicaltrials.gov/ct2/show/NCT04329832

U. S. National Library of Medicine, Clinical Trials System (2020b) Clinical study to evaluate the performance and safety of Favipiravir in COVID-19 NCT04336904. Created on 04/04/2020, Accessed on 16 Apr 2020. https://www.clinicaltrials.gov/ct2/show/ NCT04336904

U. S. National Library of Medicine, Clinical Trials System (2020c) Treatment of COVID-19 cases and chemoprophylaxis of contacts as prevention (HCQ4COV19) NCT04304053. Created on 07/04/2020, Accessed on 16 Apr 2020. https://www.clinicaltrials.gov/ct2/show/ NCT04304053 